\title{
Single embryoid body formation in a multi-well plate
}

\author{
Uthayashanker R. Ezekiel* \\ GeneProTech, Inc. \\ 9103 Lawn Avenue \\ St. Louis, MO 63144 \\ USA \\ Tel: 3149778476 \\ Fax: 3149623749 \\ E-mail: uezekiel@geneprotech.com \\ Mariappan Muthuchamy \\ Department of Systems Biology and Translational Medicine \\ Cardiovascular Research Institute \\ College of Medicine \\ Texas A and M University System Health Science Center \\ College Station, Texas 77843 \\ USA \\ Tel: 9798479251 \\ Fax: 9798624638 \\ E-mail: marim@tamu.edu
}

Jan S. Ryerse

Department of Pathology

Saint Louis University Medical Center

Saint Louis, MO 63104 USA

Tel: 3149777848

Fax: 3149778740

E-mail: ryersejs@slu.edu

\section{Rita M. Heuertz}

Department of Clinical Laboratory Science

Doisy College of Health Sciences

Saint Louis University Medical Center

Saint Louis, MO 63104 USA

Tel: 3149778610

Fax: 3149778503

E-mail: heuertzr@slu.edu

Keywords: differentiation, EB, ES, murine embryonic stem cell.

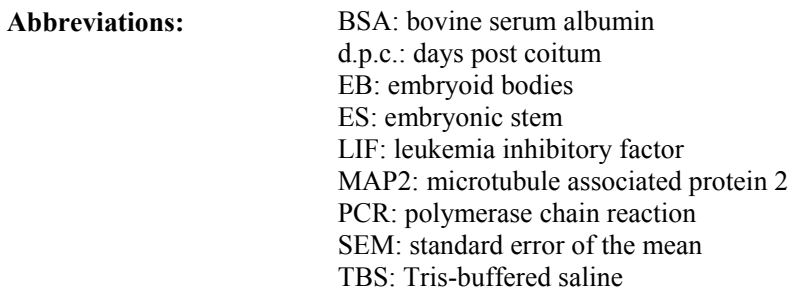

Abbreviations:

BSA: bovine serum albumin

d.p.c.: days post coitum

EB: embryoid bodies

ES: embryonic stem

LIF: leukemia inhibitory factor

MAP2: microtubule associated protein 2

PCR: polymerase chain reaction

SEM: standard error of the mean

TBS: Tris-buffered saline

Embryoid bodies (EB) formed from murine embryonic stem (ES) cells recapitulate many aspects of a developing embryo. Of specific importance, synchronous differentiation of EB recapitulates organspecific development and is achieved in culture by formation of uniformly sized EB. The method described here demonstrates a simple and cost-effective way of generating EB from murine ES cells. Single EB are formed in a multi-well plate format and large numbers of EB are generated using a 96-well multi-well plate. Uniform single-sized EB formed in the multi-well are an ideal system for screening compounds and determining differentiation effects. Since EB contain all three germ layers, they are appropriate for studying small molecule effects on differentiation of ES such as is performed in high-throughput screening protocols.

*Corresponding author 
Murine embryonic stem (ES) cells are pluripotent and derived from the inner cell mass of an early embryo. When cultured in the presence of anti-differentiation agents such as leukemia inhibitory factor (LIF) and embryonic fibroblasts, these cells maintain their pluripotency and have the ability to differentiate into any cell type of the body (Evans and Kaufman, 1981; Bain et al. 1995; Smith, 2001; Chen et al. 2003). Removal of the anti-differentiation agent causes the ES cell to spontaneously differentiate which tends to follow a reproducible temporal pattern and which, in many ways, recapitulates early embryogenesis. When ES cells are cultured in suspension without anti-differentiation agents, they form three-dimensional aggregates called embryoid bodies (EB). Within 2-4 days in suspension culture, endoderm form from the inner cell mass thereby giving rise to simple EB (Leahy et al. 1999). On approximately day 4, differentiation of columnar epithelium with a basal lamina and formation of a central cavity occurs. At this stage, the EB are called cystic embryoid bodies and bear similarity to the egg-cylinder stage of mouse embryos (Evans and Kaufman, 1981; Keller et al. 1993; $\mathrm{Ng}$ et al. 2005). Upon continued in vitro culture, EB give rise to all three germ layers (ectoderm, mesoderm, endoderm) and terminally differentiate into a wide variety of cell types, such as cardiomyocytes, hematopoietic cells, neurons and pancreatic islet cells (Risau et al. 1988; Bain et al. 1995; Smith, 2001; Dang et al. 2002; Liersch et al. 2006).

From the time of implantation and throughout gastrulation, early mammalian development is characterized by rapid cellular proliferation and migration. In mouse embryogenesis, the transitory movements of gastrulation begin shortly after implantation (6.5 days post coitum [d.p.c.]) thereby placing the endoderm, ectoderm and mesoderm in their respective positions for organogenesis. Comparison studies of murine EB developmental patterns versus murine embryonic development have been performed using gene expression and tagged reporter constructs (Xiong et al. 1998; Leahy et al. 1999; Vallier et al. 2001). Results indicate that prior to day 3, EB in suspension culture are equivalent developmentally to pregastrulation-stage embryos (4.5 - 6.5 d.p.c). Between days 3 and 5, EB contain cell types present in embryos during gastrulation (6.5 - 7.5 d.p.c.). After day 6, EB are equivalent to embryos in the stage of early organogenesis (7.5 d.p.c).

Differentiation of EB into particular cell lineages is an area of intensive study. Most of the methods utilized for EB differentiation studies involve several steps: 1) production of EB from suspension culture; 2) exposure of EB to agents designed to induce differentiation of a specific lineage; and 3) growth of EB on tissue culture plates coated with biological molecules such as gelatin or laminin (Snodgrass et al. 1992; Bain et al. 1995; Boheler et al. 2002; Dang et al. 2004). Since EB give rise to all types of cells and recapitulate events similar to those that occur during embryonic development, the use of EB to study effects of small molecules and/or biological agents is an ideal system. Indeed, there are many advantages to using EB as a model system instead of embryos or whole animals. For example, a large number of EB can be produced and used for throughput screening assays in a manner that is costeffective, especially when compared to the use of whole animals in numbers sufficient for screening purposes.

For the development of a system applicable to throughput screening formats, EB have been chosen as the model system for the determination of differentiation effects by biological agents. Since the goal of this study was to

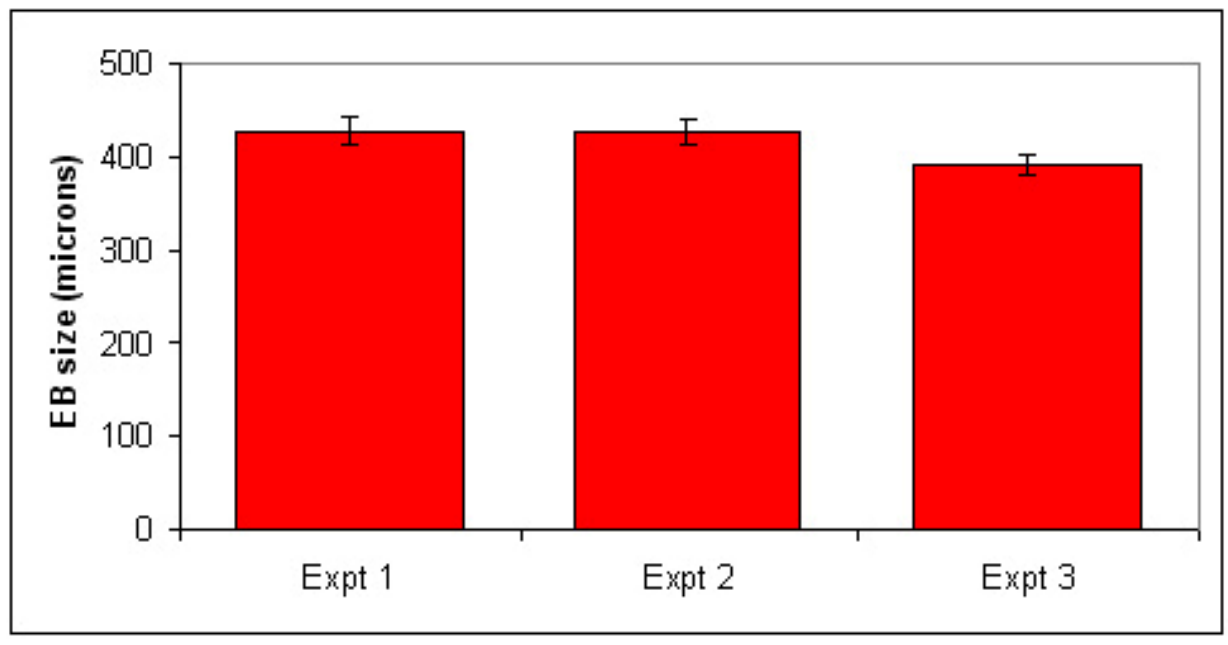

Figure 1. Distribution of a single EB into a microwell. For each experiment, individual EB sizes were measured using a micrometer and phase contrast microscopy. Results were from three independent experiments and are displayed as mean micron diameter \pm SEM. EB mean average diameter \pm SEM in experiment one was $428 \pm 14$ microns $(n=20$ randomly picked EB), in experiment two was $427 \pm$ 14 microns $(n=20$ randomly picked EB) and in experiment 3 was $392 \pm 11$ microns $(n=17$ randomly picked EB). 


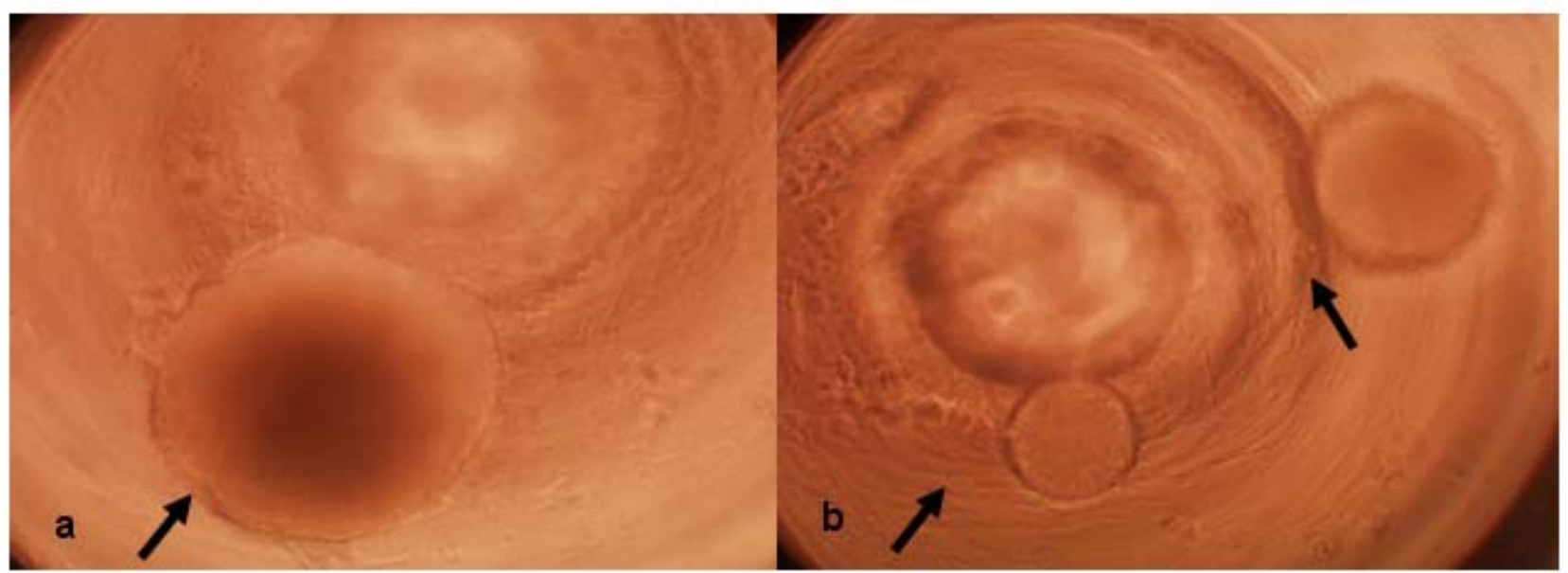

Figure 2. Single EB in 96-well format polycarbonate PCR plate. Results displayed in a and a are phase contrast microscopy using the same magnification.

(a) Single $E B$ in a well. A single $E B$ is present in the microwell and the single $E B$ is of typical size. (b)EB with dissimilar size and number. Two EB are present in the microwell and the two EB are of dissimilar sizes. Additionally, sizes of the two EB are dissimilar from the single EB displayed in a arrows point to the EB.

identify EB as a model system for differentiation studies by throughput methodologies, it was essential to develop a throughput-screening protocol using EB. For screening purposes, uniform-sized EB have been shown to have synchronous differentiation potentials $(\mathrm{Ng}$ et al. 2005). Therefore, a goal of this study was to determine uniformity of size of EB for use in throughput screen methodologies.

Current techniques for EB studies include hanging drop and suspension methodologies (Dang et al. 2002; Gerecht-Nir et al. 2004). The suspension culture method gives rise to non uniformly sized cells (Brook and Gardner, 1997; Dang et al. 2002; Gerecht-Nir et al. 2004). The hanging drop method provides uniform sizes of EB; however, this technique is challenging to perform and not amenable to throughput screening strategies (Dang et al. 2002; Kurosawa et al. 2003). Current methods that ensure uniform sizes of EB in a screening format are not simple procedures and require the use of sophisticated flow sorting equipment (Fernández et al. 2005). Additionally, to dispense single EB into wells by currently available automated methods is not cost-effective. For these reasons, a simple, cost-effective method in which EB are generated of uniform size and singularity is herein described. Additionally, the EB are dispensable into micro-wells in a manner that enables throughput methodologies for screening purposes. The use of single EB in a multi-well format is ideal because theoretically, one can consider a single EB in a well as an equivalent to a developing embryo, or in other words, a single EB is a developing organism in a microwell. The method described here allows screening and testing of biochemical compounds, biological agents and infectious organisms as well as allowing for toxicity evaluation.

\section{MATERIALS AND METHODS}

ES cells derived from $129 / \mathrm{SvJ}$ mice were maintained in culture on a layer of feeder cells consisting of mitomycin C-treated primary mouse embryonic fibroblasts (MEF) (Brook and Gardner, 1997). The ES cells were cultured in ES medium (DMEM supplemented with $15 \%$ serum (Hyclone, Logan, UT), 1000 units/ml LIF (ESGRO from Chemicon, Temecula, CA), $1 \mathrm{mM}$ sodium pyruvate, $1 \mathrm{mM}$ nonessential amino acids, $0.1 \mathrm{mM}$ 2-mercaptoethanol, 25 units $/ \mathrm{ml}$ penicillin and $25 \mu \mathrm{g} / \mathrm{ml}$ streptomycin). The ES cells were plated onto prepared feeder layers with freshly prepared ES medium and incubated in a humidified chamber $\left(37^{\circ} \mathrm{C}, 5 \% \mathrm{CO}_{2}\right)$. Media was changed every other day for maintenance of the ES cells.

As a pre-differentiation step, feeder cells were removed from the ES cell culture. To accomplish feeder cell removal, ES cells were passaged three times onto $0.1 \%$ gelatin-coated tissue culture plates using the following protocol. The cells were trysinized (5 min) and trypsin was inactivated by addition of an equal volume of media followed by incubation in a humidified chamber (15 $\mathrm{min}$, $\left.37^{\circ} \mathrm{C}, 5 \% \mathrm{CO}_{2}\right)$. After incubation, most feeder cells remained attached to the plates whereas the ES cells were in solution. ES cells were transferred to $0.1 \%$ gelatin-coated tissue culture plates which supported ES cell growth. The presence/absence of feeder cells was microscopically checked. This process was repeated a second and a third time. After the third passage, all ES cells were completely devoid of feeder cells.

For differentiation, the ES cells on gelatin-coated tissue culture plates were washed with phosphate buffered saline (PBS), trypsinized, dissociated into single cells and 
suspended in differentiation medium (same formulation as ES medium except devoid of LIF). ES cells were counted using a hemocytometer and number of surviving cells was determined by trypan blue dye exclusion $(>99 \%$ cell viability). The final cell number was adjusted to $5 \times 10^{3}$ cells $/ \mathrm{ml}$.

To achieve single EB with uniformity of size, 1,000 ES cells in a $200 \mu \mathrm{l}$ volume were dispensed into sterile 96-well polyvinyl carbonate polymerase chain reaction (PCR), plates (Greiner Bio-One Inc, Longwood, FL), tapped gently and incubated $\left(37^{\circ} \mathrm{C}, 5 \% \mathrm{CO}_{2}\right)$. After 4 days, the number of $\mathrm{EB} /$ well was assessed by observation using light microscopy. The EB grown in polyvinyl carbonate PCR plates or other similar plastic surfaces did not attach and the 4-day old EB grown in these plates were called 4-day suspension EB (4-dsEB). To obtain mean diameter values, EB were randomly picked and diameter measured microscopically. For differentiation studies, EB grown for 4 days were transferred from a $96-$-well PCR plate using a multi-channel pipette fitted with tips of broad pore size into a 96-well tissue culture plate coated with $0.1 \%$ gelatin. For a beating cardiac muscle phenotype, EB in plates were visually observed for foci of beating activity.

For neuron differentiation, 4-dsEB were transferred to gelatin $(0.1 \%)$-coated 96-well tissue culture plates and exposed to $1 \mu \mathrm{M}$ retinoic acid (Bain et al. 1995). After the $4^{\text {th }}$ day of retinoic acid exposure, the wells received differentiation medium (formulation stated above). To verify EB differentiation, EB were dissociated and grown on coverslips coated with $0.1 \%$ gelatin in 6-well microplates, allowed to differentiate for 12 days and subjected to immunocytochemistry. To identify post-mitotic neurons, expression of a neuronal antigenic marker, microtubule associated neuron cytoskeletal protein (MAP2), was assessed by immunocytochemistry using antibody reactivity to MAP2 $[\mathrm{a}+\mathrm{b}]$ (Sigma Chemical, St. Louis, $\mathrm{MO})$. For in situ immunofluorescence, cells grown on gelatin-coated coverslips were rinsed with Tris-buffered saline (TBS $50 \mathrm{mM}$ Tris- $\mathrm{HCl}, \mathrm{pH} 7.5,150 \mathrm{mM} \mathrm{NaCl}$ ) and fixed with $4 \%$ paraformaldehyde in TBS for $10 \mathrm{~min}$. Cells were washed with TBS and incubated (overnight, $4^{\circ} \mathrm{C}$ ) with primary anti-MAP2 $[\mathrm{a}+\mathrm{b}]$ monoclonal antibody (Sigma Chemical) in $1 \%$ bovine serum albumin (BSA) and $0.05 \%$ Triton X100. Cells were washed with TBS and incubated with Oregon Green-conjugated secondary antibody (Molecular Probes, Eugene, OR) in TBS, $0.05 \%$ Triton $\mathrm{X} 100$ and $1 \%$ BSA for $30 \mathrm{~min}$. Cells were visualized using fluorescent microscopy.

Results were displayed as means \pm standard error of the mean (SEM). Statistical analyses were by an unpaired, twotailed Students t-test or by the Kruskal-Wallis test followed by Dunn's multiple comparisons test when indicated.

\section{RESULTS}

ES cells in 96-well PCR microplates were allowed to form EB. On the fourth day, the 4-dsEB were assessed as to number of EB per microwell. Table 1 shows results of four independent experiments to determine number of $\mathrm{EB} / \mathrm{well}$ using the above-described method. All microwells contained either one or two EB per well with the predominant number of wells containing a single EB. Microwells containing more than two EB were never observed. In the four independent experiments, $92 \%, 97 \%$, $95 \%$ and $93 \%$ (mean 94\%) of the wells in 96-well multiwell plates contained a single EB. Two EB per well were observed $6 \%$ of the time $(n=4)$.

To obtain a mean value for EB size from the 4-dsEB, single EB were randomly picked and EB diameters measured. Results from three independent experiments are displayed

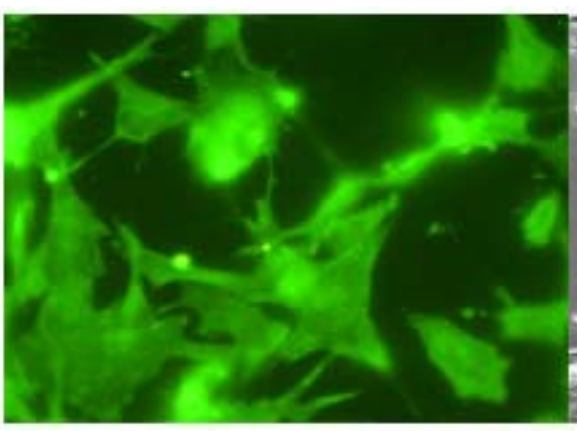

a

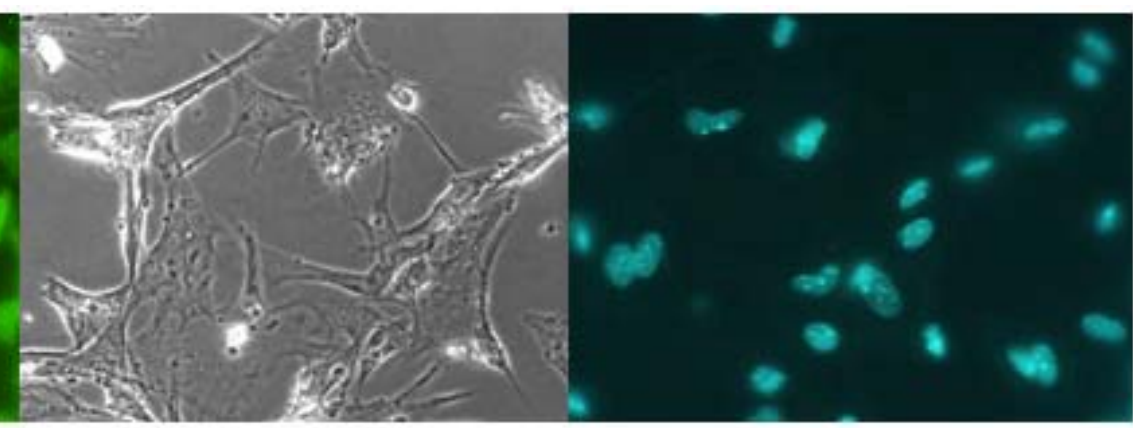

b
C

Figure 3. Characterization of ES cells differentiated into neurons on day 12 of differentiation.

(a) Cells stained with neuronal marker. Cells show indirect immunofluorescence of Oregon Green-conjugated antibody against the neuronal cell marker, cytoskeletal protein MAP2 [a+b]. Cells that fluoresce are MAP2 positive and display the neuronal cell phenotype.

(b) Cell showing neuronal morphology. The same field of cells shown in (a) are displayed using bright field microscopy. Characteristic neuronal morphology is evident.

(c) Cells stained with DAPI showing the nucleus. The same field of cells shown in (a) and (b) is displayed as DAPI-staining of the nucleus. 
in Figure 1. The mean average diameter \pm SEM of randomly picked EB in experiment one was $428 \pm 14$ microns $(\mathrm{n}=20)$. In the second experiment, mean average diameter \pm SEM of randomly picked EB was $427 \pm 14$ microns $(n=20)$ and in experiment 3 , mean average diameter \pm SEM of randomly picked EB was $392 \pm 11$ microns $(n=17)$. No statistically significant difference was identified among the three experiments. Mean average diameter of all three experiments (57 randomly picked EB) was 416 microns.

Photomicrographs of EB in micro-well plates are displayed in Figure 2a single EB in micro-well format is displayed in Figure 2a and a well containing more than one EB is shown in Figure 2b. The EB were attached at either central or peripheral locations in the micro-well. As stated above, the most frequent observation was a single $\mathrm{EB} /$ well in this format. Rarely, two EB/well were evident. Every time two $\mathrm{EB} /$ well were present, the EB were either uniform or dissimilar in size by this method.

Use of single $\mathrm{EB} /$ well in differentiation studies was demonstrated. It has been established in the literature that EB give rise to differentiated cardiac muscle cells (Doetschman et al. 1993; Muthuchamy et al. 1993; Kurosawa et al. 2003). Of importance, transfer of EB by the method reported here did not result in any mechanical injury-induced loss of EB as evidenced by the presence of intact cells of similar spherical morphology immediately after transfer as before manipulation. The EB in each well were periodically observed microscopically for generation of a unique characteristic, specifically of a beating cardiac muscle phenotype in the population of cells derived from the EB. A result was considered positive if an EB containing foci of a beating cardiac muscle was observed. A few EB showed foci of beating cells on day 3 of plating; however, by day 7, all EB in the wells were observed to have foci of beating cardiac muscle cells.

In addition to cardiac-specific differentiation, use of the method described here allowed for the differentiation of EB into neuronal cells. The presence of neurite growth indicated that the EB in multi-well format had the ability to form neuronal cells. To verify the cells as neurons, indirect immunofluorescence for the presence of the MAP2 neuronal cell marker was performed on day 12differentiated cells. Anti-MAP2 immunofluorescence positive cells with a typical neuronal morphology are shown in Figure 3a. Figure $3 \mathrm{~b}$ displays the same field of cells as Figure $3 \mathrm{a}$ but with the use of bright field microscopy. Figure $3 \mathrm{c}$ shows results of the same field of cells (as displayed in Figures 3a and Figure 3b) but with DAPI-staining of the nucleus. The results indicate that on day 12 of differentiation, neuronal cells are present.

\section{DISCUSSION}

EB formed from murine ES cells recapitulate many aspects of a developing embryo. Synchronous differentiation of EB is achieved by formation of uniformly sized $\mathrm{EB}(\mathrm{Ng}$ et al. 2005). The method reported here is cost-effective and easy to perform. Additionally, a large number of $\mathrm{EB}$ can be generated using the 96-well PCR plate method followed by transfer of the EB to 96-well tissue culture plates for differentiation and screening purposes. On average, 94\% of the wells have a single EB with mean average diameter of 415 microns. The EB formed by this method differentiate into different cell types with specific differentiation into beating cardiomyocytes and neurons being assessed in this study.

The differentiation of ES cells into various lineages is an area of intense study because of the direct applicability of ES cells to the field of regenerative medicine (Smith, 2001; Ding and Schultz, 2004; Kumar et al. 2005). The in vitro

Table 1. Most wells in 96-well microplate format support growth of a single embryoid body.

\begin{tabular}{|l|c|c|c|c|}
\hline & \multicolumn{2}{|c|}{$\begin{array}{c}\text { One Embryoid Body } \\
\text { in Microwell }\end{array}$} & \multicolumn{2}{c|}{$\begin{array}{c}\text { Two Embryoid Bodies } \\
\text { in Microwell }\end{array}$} \\
\hline & Raw Data & $\%$ Total & Raw Data & $\%$ Total \\
\hline Expt 1 & $88 / 96$ wells & $92 \%$ & $9 / 96$ wells & $9 \%$ \\
\hline Expt 2 & $93 / 96$ wells & $97 \%$ & $3 / 96$ wells & $3 \%$ \\
\hline Expt 3 & $91 / 96$ wells & $95 \%$ & $5 / 96$ wells & $5 \%$ \\
\hline Expt 4 & $89 / 96$ wells & $93 \%$ & $7 / 96$ wells & $7 \%$ \\
\hline & $361 / 384$ wells & $94 \% *$ & $24 / 384$ wells & $6 \%$ \\
\hline$*=0<0.001$ of 1 EB/well versus 2 EB/well. & & & \\
\hline
\end{tabular}


differentiation capabilities of ES cells allow their use as a model system for the study of developmental potential as well as their use as a valuable reagent for stem cell therapeutic approaches. Several methodologies exist by which EB are formed and differentiated into different cell types. The typical method of EB formation is by removal of LIF and the feeder cell layer followed by growth in bacterial grade Petri dishes of EB in suspension. While this is a commonly used method, EB formation occurs but with great variability in EB shape and EB size (Doevendans et al. 2000; Dang et al. 2002). Several technologies for largescale EB growth have been reported with examples being suspension cultures in bioreactors and encapsulation of ES cells in alginate microbeads or agarose capsules (Magyar et al. 2001; Dang et al. 2002; Gerecht-Nir et al. 2004; Konno et al. 2005). EB formation in the presence of feeder cells, specifically STO cells, and subsequent differentiation of these EB has been reported (Zhou et al. 2005). Currently, uniform-size single EB are usually generated by a hanging drop method in which the cells are allowed to aggregate in a hanging drop. This method is laborious and a paramount task is the acquisition of a sufficient number of EB for screening purposes (Kurosawa et al. 2003). The uniformity of size observed in EB formed by the hanging drop method has been suggested to be important for subsequent EB differentiation since endogenously produced LIF may exert an autocrine or paracrine function even though exogenous LIF is removed (Ansell and Hole, 2000).

In the hanging drop method, an EB suspension containing approximately 300 cells in a small volume $(10 \mu \mathrm{l})$ are placed onto the lid of a Petri plate. The Petri plate lid is placed onto its base with a swift action so as to not disperse the drop. The plate is kept in a humidified incubator $(7.5 \%$ $\mathrm{CO}_{2}$ in air, $48 \mathrm{hrs}$ ). After two days, the cells have settled into the meniscus of the hanging drop and have formed smooth, spherical aggregates of uniform size. The EB are then pooled and used for differentiation studies. Since all the EB are present in the small droplet, an ever-present problem with the hanging drop method is that there is no easy way to subject individual EB to different biological agents. In the method described here, the EB settle to the bottom of the microwell in a multi-well plate. Slight tapping of the multi-well plate causes the EB to accumulate at the base of the well which then allows for single uniform-sized EB to be dispensed into the individual wells. Additionally, several polyvinyl carbonate PCR plates can be used to generate large numbers of single, uniform-sized EB which can then be transferred to multi-well tissue culture plates for subsequent differentiation and screening purposes. Herein we show that EB formed by this method are efficiently differentiated into cardiomyocytes and neurons. The formation of single, uniform-size EB in a multi-well format provides an efficient in vitro system to screen for effects of small molecules and bioagents and is an alternative to embryo and live animal studies. Using this in vitro system, it is possible to screen many compounds efficiently. Once a candidate molecule is identified, a thorough study can be performed to define how the molecule exerts its effect using throughput technology.

To streamline throughput screening studies, ES cell lines containing a reporter construct under the control of a specific promoter in the form of a promoter-reporter cassette is of great interest. Reporters expressed only in lineages where the promoter is functional is an initial approach worth assessment. The differentiated cell type will be identifiable by analysis of reporter gene expression. Screening compounds may be added to EB and analysis of reporter expression would show whether the small molecule inhibits or augments differentiation (or other function) or has no effect on cell lineage differentiation. By creation of a panel of lineage-specific promoter-reporter ES cell lines, screening of EB from these cell lines has the potential to provide an excellent system for testing differentiation effects of small molecules. Currently, work is in progress to engineer ES cells lines useful for these types of aforementioned screening purposes. Formation of ES cells giving rise to uniform-sized, single EB is crucial for the success of this screening strategy. Information gleaned from this panel will be useful in determining whether a particular small molecule is toxic to the embryo or is a modulator of development of a particular cell lineage. Differentiation of ES cells into different cell lineages is of great importance since the potential exists for application of discovered technologies as therapeutics for regenerative medicine.

\section{REFERENCES}

ANSELL, John and HOLE, Nicholas. Haematopoietic differentiation of embryonic stem cells. In: DALLAMN, Margaret J. and LAMB, Jonathan R. eds. Haematopoietic and Lymphoid Cell Culture. Cambridge University Press, 2000, p. 5-23.

BAIN, Gerard; KITCHENS, Daniel; YAO, Min; HUETTNER, James E. and GOTTLIEB, David I. Embryonic stem cells express neuronal properties in vitro. Developmental Biology, April 1995, vol. 168, no. 2, p. 342357.

BOHELER, Kenneth R.; CZYZ, Jaroslaw; TWEEDIE, David; YANG, Huang-Tian; ANISIMOV, Sergey V. and WOBUS, Anna M. Differentiation of pluripotent embryonic stem cells into cardiomyocytes. Circulation Research, August 2002, vol. 91, no. 3, p. 189-201.

BROOK, F.A. and GARDNER, R.L. The origin and efficient derivation of embryonic stem cells in the mouse. Proceedings of the National Academy of Sciences of the United States of America, May 1997, vol. 94, no. 11, p. 5709-5712.

CHEN, Dong; LEWIS, Rachel L. and KAUFMAN, Dan S. Mouse and human embryonic stem cell models of 
hematopoiesis: past, present, and future. BioTechniques, December 2003, vol. 35, no. 6, p. 1253-1261.

DANG, Stephen M.; GERECHT-NIR, Sharon; CHEN, Jinny; ITSKOVITZ-ELDOR, Joseph and ZANDSTRA, Peter W. Controlled, scalable embryonic stem cell differentiation culture. Stem Cells, December 2004, vol. 22, no. 3, p. 275-282.

DANG, Stephen M.; KYBA, Michael; PERLINGEIRO, Rita; DALEY, George Q. and ZANDSTRA, Peter W. Efficiency of embryoid body formation and hematopoietic development from embryonic stem cells in different culture systems. Biotechnology and Bioengineering, May 2002, vol. 78 , no. 4 , p. 442-453.

DING, Sheng and SCHULTZ, Peter G. A role for chemistry in stem cell biology. Nature Biotechnology, July 2004, vol. 22, no. 7, p. 833-840.

DOETSCHMAN, T.; SHULL, M.; KIER, A. and COFFIN, J.D. Embryonic stem cell model systems for vascular morphogenesis and cardiac disorders. Hypertension, October 1993, vol. 22, no. 4, p. 618-629.

DOEVENDANS, Pieter A.; KUBALAK, Steven W.; AN, Rui-Hai; BECKER, David K.; CHIEN, Kenneth R. and KASS, Robert S. Differentiation of cardiomyocytes in floating embryoid bodies is comparable to fetal cardiomyocytes. Journal of Molecular and Cellular Cardiology, May 2000, vol. 32, no. 5, p. 839-851.

EVANS, M.J. and KAUFMAN, M.H. Establishment in culture of pluripotential cells from mouse embryos. Nature, July 1981, vol. 292, no. 5819, p. 154-156.

FERNÁNDEZ, Luis A.; HATCH, Eric W.; ARMANN, Barbara; ODORICO, Jon S.; HULLETT, Debra A.; SOLLINGER, Hans $\mathrm{W}$. and HANSON, Matthew S. Validation of large particle flow cytometry for the analysis and sorting of intact pancreatic islets. Transplantation, September 2005, vol. 80, no. 6, p. 729-737.

GERECHT-NIR, Sharon; COHEN, Smadar; ZISKIND, Anna and ITSKOVITZ-ELDOR, Joseph. Threedimensional porous alginate scaffolds provide a conducive environment for generation of well-vascularized embryoid bodies from human embryonic stem cells. Biotechnology and Bioengineering, November 2004, vol. 88, no. 3, p. 313320 .

KELLER, G.; KENNEDY, M.; PAPAYANNOPOULOU, T. and WILES, M.V. Hematopoietic commitment during embryonic stem cell differentiation in culture. Molecular and Cellular Biology, January 1993, vol. 13, no. 1, p. 473486.

KONNO, Tomohiro; AKITA, Kunihiko; KURITA, Kimio and ITO, Yoshihiro. Formation of embryoid bodies by mouse embryonic stem cells on plastic surfaces. Journal of
Bioscience and Bioengineering, July 2005, vol. 100, no. 1, p. 88-93.

KUMAR, Dinender; KAMP, Timothy J. and LEWINTER, Martin M. Embryonic stem cells: differentiation into cardiomyocytes and potential for heart repair and regeneration. Coronary Artery Disease, March 2005, vol. 16, no. 2, p. 111-116.

KUROSAWA, Hiroshi; IMAMURA, Tetsuya; KOIKE, Mikiko; SASAKI, Katsunori and AMANO, Yoshifumi. A simple method for forming embryoid body from mouse embryonic stem cells. Journal of Bioscience and Bioengineering, July 2003, vol. 96, no. 4, p. 409-411.

LEAHY, Amy; XIONG, Jing-Wei; KUHNERT, Frank and STUHLMANN, Heidi. Use of developmental marker genes to define temporal and spatial patterns of differentiation during embryoid body formation. Journal of Experimental Zoology, June 1999, vol. 284, no. 1, p. 67-81.

LIERSCH, Ruediger; NAY, Filip; LU, Lingge and DETMAR, Michael. Induction of lymphatic endothelial cell differentiation in embryoid bodies. Blood, February 2006, vol. 107 , no. 3 , p. 1214-1216.

MAGYAR, Josef P.; NEMIR, Mohamed; EHLER, Elisabeth; SUTER, Nicolai; PERRIARD, Jean-Claude and EPPENBERGER, Hans M. Mass production of embryoid bodies in microbeads. Annals of the New York Academy of Sciences, November 2001, vol. 944, p. 135-143.

MUTHUCHAMY, M.; PAJAK, L.; HOWLES, P.; DOETSCHMAN, $T$. and WIECZOREK, D.F. Developmental analysis of tropomyosin gene expression in embryonic stem cells and mouse embryos. Molecular and Cellular Biology, June 1993, vol. 13, no. 6, p. 3311-3323.

NG, Elizabeth S.; DAVIS, Richard P.; AZZOLA, Lisa; STANLEY, Edouard G. and ELEFANTY, Andrew G. Forced aggregation of defined numbers of human embryonic stem cells into embryoid bodies fosters robust, reproducible hematopoietic differentiation. Commentary. Blood, September 2005, vol. 106, no. 5, p. 1601-1603.

RISAU, W.; SARIOLA, H.; ZERWES, H.G.; SASSE, J.; EKBLOM, P.; KEMLER, R. and DOETSCHMAN, T. Vasculogenesis and angiogenesis in embryonic-stem-cellderived embryoid bodies. Development, March 1988, vol. 102, no. 3, p. 471-478.

SMITH, Austin G. Embryo-derived stem cells: of mice and men. Annual Review of Cell and Developmental Biology, November 2001, vol. 17, p. 435-462.

SNODGRASS, H. Ralph; SCHMITT, Regina M. and BRUYNS, Eddy. Embryonic stem cells and in vitro hematopoiesis. Journal of Cellular Biochemistry, July 1992, vol. 49, no. 3, p. 225-230. 
Ezekiel, U. et al.

VALLIER, Ludovic; MANCIP, Jimmy; MARKOSSIAN, Suzy; LUKASZEWICZ, Agnès; DEHAY, Colette; METZGER, Daniel; CHAMBON, Pierre; SAMARUT, Jacques and SAVATIER, Pierre. An efficient system for conditional gene expression in embryonic stem cells and in their in vitro and in vivo differentiated derivatives. Proceedings of the National Academy of Sciences of the United States of America, February 2001, vol. 98, no. 5, p. 2467-2472.

XIONG, Jing-Wei; BATTAGLINO, Ricardo; LEAHY, Amy and STUHLMANN, Heidi. Large-scale screening for developmental genes in embryonic stem cells and embryoid bodies using retroviral entrapment vectors. Developmental Dynamics, June 1998, vol. 212, no. 2, p. 181-197.

ZHOU, Qing-Jun; SHAO, Jian-Zhong; XIANG, Li-Xin; HU, Ruo-Zhen; LU, Yong-Liang; YAO, Hang and DAI, LiCheng. Generation of embryoid bodies from mouse embryonic stem cells cultured on STO feeder cells. Cell Biology International, September 2005, vol. 29, no. 9, p. 817-825. 